\title{
Search for a charged Higgs boson decaying to a top and a bottom quarks in ATLAS
}

\author{
Lluïsa-Maria Mir*† \\ IFAE - Institut de Física d'Altes Energies \\ E-mail: mireifae.es
}

\begin{abstract}
A search for charged Higgs bosons decaying to a top quark and a bottom quark is presented. The analysis uses $13.2 \mathrm{fb}^{-1}$ of $p p$ collision data at a centre-of-mass energy of $13 \mathrm{TeV}$ collected with the ATLAS detector at the Large Hadron Collider. No significant excess above the backgroundonly hypothesis is observed and upper limits on the production cross section times the decay branching fraction are set.
\end{abstract}

Prospects for Charged Higgs Discovery at Colliders

3-6 October 2016

Uppsala, Sweden

* Speaker.

${ }^{\dagger}$ On behalf of the ATLAS Collaboration 
The Standard Model of particle physics (SM) does not comprise any charged scalar bosons, but many theories beyond the Standard Model (BSM) include an extended Higgs sector with at least one pair of charged Higgs bosons. ${ }^{1}$ The discovery of a charged scalar boson would thus be a clear indication of BSM physical phenomena. The production mechanisms and decay modes of charged Higgs bosons depend on their mass, and for a charged Higgs boson heavier than the topquark mass the dominant production mode is in association with a top quark. The decay depends on the parameters of the model, and for two-Higgs-doublet models in the limit of $\cos (\beta-\alpha) \approx 0^{2}$ the dominant decay mode is $H^{+} \rightarrow t b$.

In these proceedings, a search for $H^{+}$production in $p p$ collisions at a centre-of-mass energy of $13 \mathrm{TeV}$ collected with the ATLAS detector [1] at the Large Hadron Collider (LHC) is presented. The analysis uses $13.2 \mathrm{fb}^{-1}$ of $p p$ collision data recorded during 2015 and part of 2016, and targets $\mathrm{H}^{+}$decays to a top quark and a bottom quark [2]. The events were recorded using single-lepton triggers and only events with exactly one lepton (electron or muon) in the final state are considered. The lepton must be associated with the lepton trigger and have $p_{T}$ above $25 \mathrm{GeV}$. In addition, events must contain at least four jets with a $p_{T}$ larger than $25 \mathrm{GeV}$, of which at least two must be $b$-tagged. The $b$-tagging algorithm has an efficiency of $70 \%$ for $b$-jets in $t \bar{t}$ events, and $c$-jet and light-jet rejection factors of 8 and 440, respectively. After event selection, $t \bar{t}$ events are the main background, which is simulated using the Powheg-Box v2 next-to-leading order (NLO) in QCD generator with the CT10 parton density function (PDF) set. The $t b H^{+}$process is modelled by Madgraph5_aMC@NLO, which is a NLO in QCD generator, using the four-flavour scheme, with the NNPDF2.3 PDF set. Details about the generators can be found in reference [2].

To take advantage of the larger jet and $b$-jet multiplicities of events with a $H^{+} \rightarrow t b$ decay with respect to the SM background, events passing the event selection are categorised into separate regions according to their jet and $b$-jet multiplicities. Signal regions (SR) are those where $H^{+} \rightarrow t b$ events are enhanced with respect to the backgrounds, whereas the remaining regions are taken as control regions (CR) and are designed to measure backgrounds and to constrain systematic uncertainties. Four CR $(4 \mathrm{j} 2 \mathrm{~b}, 4 \mathrm{j} \geq 3 \mathrm{~b}, 5 \mathrm{j} 2 \mathrm{~b}$ and $\geq 6 \mathrm{j} 2 \mathrm{~b})$ and four $\mathrm{SR}(5 \mathrm{j} 3 \mathrm{~b}, 5 \mathrm{j} \geq 4 \mathrm{~b}, \geq 6 \mathrm{j} 3 \mathrm{~b}$ and $\geq 6 \mathrm{j} \geq 4 \mathrm{~b}$ ), where $X \mathrm{j} Y \mathrm{~b}$ means that $X$ jets are found in the event and among them $Y$ are $b$-tagged, are used in the analysis.

Different discriminant variables to separate signal from background are used depending on the event category. The variable chosen in the CR is the scalar sum of the $p_{T}$ of the selected jets, $H_{T}^{\text {had }}$, whereas the output of boosted decision trees (BDTs) is used in the SR. The BDTs are trained separately at each value of the $H^{+}$mass. Twelve kinematical variables [2] enter the BDTs, among them the leading jet transverse momentum, the scalar sum of transverse energies of all jets, or the average angular separation of all $b b$ pairs, but no reconstruction of the charged Higgs boson kinematics has been attempted. This set of variables provides the best signal-background separation across all $\mathrm{H}^{+}$mass hypotheses. Figure 1 shows the expected BDT output distributions for signal and background for two values of the $H^{+}$mass hypothesis.

A binned maximum-likelihood fit to the data is performed simultaneously in all categories.

\footnotetext{
${ }^{1}$ For simplicity, in the following, charged Higgs bosons are denoted $\mathrm{H}^{+}$, with the charge-conjugate $\mathrm{H}^{-}$always implied.

${ }^{2} \tan \beta$ is the ratio of the vacuum expectation values of the two Higgs doublets, and $\alpha$ is the mixing angle between the CP-even Higgs bosons.
} 

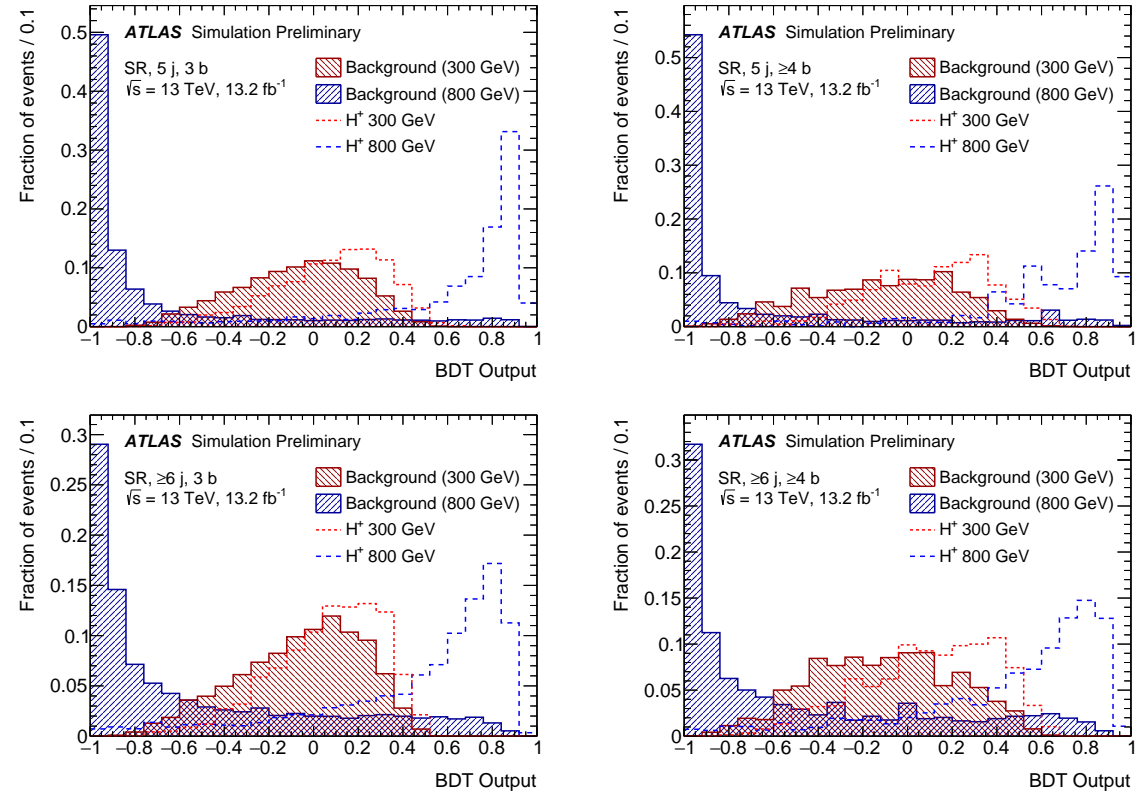

Figure 1: Expected BDT output distribution for SM backgrounds and for two $\mathrm{H}^{+}$signal mass hypotheses ( 300 and $800 \mathrm{GeV}$ ), in the four SR: $5 \mathrm{j} 3 \mathrm{~b}$ (top left), $5 \mathrm{j} \geq 4 \mathrm{~b}$ (top right), $\geq 6 \mathrm{j} 3 \mathrm{~b}$ (bottom left) and $\geq 6 \mathrm{j} \geq 4 \mathrm{~b}$ (bottom right). All distributions are normalised to unity [2].

The inputs to the fit are the distributions of $H_{T}^{\text {had }}$ in the CR and the BDT outputs in the SR. Each mass hypothesis is tested separately. Figure 2 shows the selected data compared with the SM background expectation before and after the fit.

The procedures used to quantify the agreement with the background-only hypothesis and to determine exclusions limits are based on the profile likelihood ratio test. The parameter of interest is the product of the production cross section $\sigma\left(p p \rightarrow t b H^{+}\right)$and the branching fraction $\operatorname{BR}\left(H^{+} \rightarrow t b\right)$, referred to as the signal strength $\mu$. The normalisations of the $t \bar{t}+\geq 1 b$ and $t \bar{t}+\geq 1 c$ background components are also taken as free-floating parameters in the fit. All systematic uncertainties are implemented as nuisance parameters with log-normal constraint terms. There are about 150 nuisance parameters considered in the fit. A summary of the systematic uncertainties is given in table 1.

The modified frequentist method (CLs) [3] and asymptotic formulae [4] are used to calculate upper limits on $\sigma\left(p p \rightarrow H^{+}\right) \times \mathrm{BR}\left(H^{+} \rightarrow t b\right)$. The 95\% confidence level (CL) upper limits are presented in Figure 3. The mass hypotheses are tested in $50 \mathrm{GeV}$ steps between 300 and $400 \mathrm{GeV}$ and $100 \mathrm{GeV}$ steps between 400 and $1000 \mathrm{GeV}$. The observed (expected) 95\% CL upper limits on $\sigma\left(p p \rightarrow H^{+}\right) \times \mathrm{BR}\left(H^{+} \rightarrow t b\right)$ range from $\sigma \times \mathrm{BR}=1.09(1.45) \mathrm{pb}$ at $m_{H^{+}}=300 \mathrm{GeV}$ to $\sigma \times \mathrm{BR}=0.18(0.17) \mathrm{pb}$ at $m_{H^{+}}=1000 \mathrm{GeV}$. The compatibility of the data with the SM hypothesis is quantified with the $p_{0}$ value. The smallest $p_{0}$ value is 0.017 , found at $600 \mathrm{GeV}$, corresponding to 2.1 standard deviations.

Figure 3 also shows the limits set on $\tan \beta$ for the $m_{h}^{\bmod -}$ scenario of the MSSM [5, 6, 7]. For $H^{+}$masses of $300-855 \mathrm{GeV}$ some values of $\tan \beta$ in the range $0.5-1.7$ are excluded; the expected 

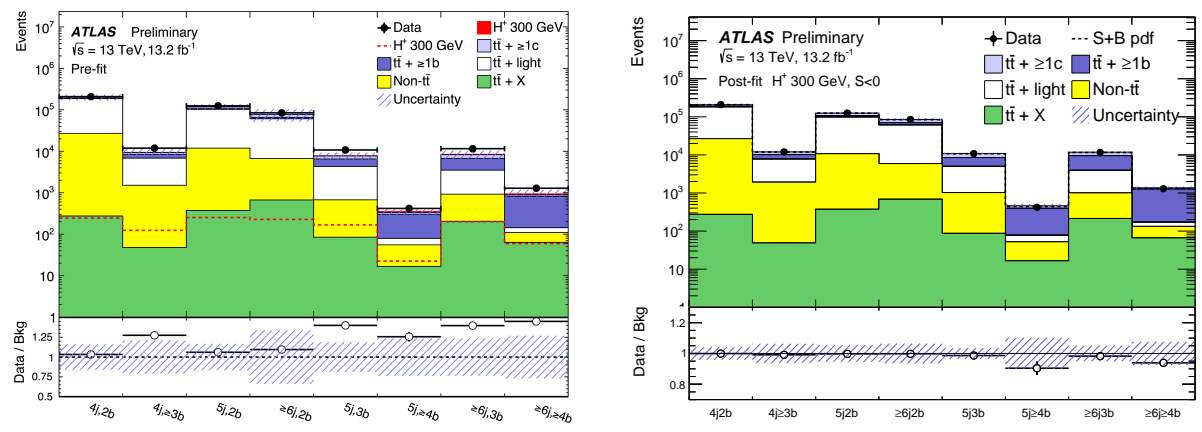

Figure 2: Comparison of predicted and observed event yields before (left) and after (right) the fit to the data under the background-plus-signal hypothesis with an $H^{+}$signal mass of $300 \mathrm{GeV}$, as an example. $t \bar{t}+X$ includes contributions from $t \bar{t}+W, t \bar{t}+Z$ and $t \bar{t}+H$. The fitted signal is slightly negative $(\mathrm{S}<0)$ and therefore, while included, is not explicitly displayed. The lower panels display the ratio of the data to the total predicted background [2].

\begin{tabular}{|l|cc|cc|}
\hline Uncertainty Source & \multicolumn{2}{|c|}{$\Delta \mu\left(H_{300}^{+}\right)$} & \multicolumn{2}{|c|}{$\Delta \mu\left(H_{800}^{+}\right)$} \\
\hline \hline$t \bar{t}+\geq 1 b$ modelling & +0.53 & -0.53 & +0.07 & -0.07 \\
Jet flavour tagging & +0.30 & -0.29 & +0.07 & -0.07 \\
$t \bar{t}+\geq 1 c$ modelling & +0.23 & -0.22 & +0.03 & -0.03 \\
Background model statistics & +0.19 & -0.19 & +0.05 & -0.05 \\
Jet energy scale and resolution & +0.18 & -0.17 & +0.03 & -0.03 \\
$t \bar{t}+$ light modelling & +0.16 & -0.16 & +0.03 & -0.03 \\
Other background modelling & +0.15 & -0.14 & +0.03 & -0.03 \\
Jet-vertex association, pileup modelling & +0.12 & -0.11 & +0.01 & -0.01 \\
$t \bar{t}+\geq 1 c$ modelling & +0.23 & -0.22 & +0.03 & -0.03 \\
Background model statistics & +0.19 & -0.19 & +0.05 & -0.05 \\
Jet energy scale and resolution & +0.18 & -0.17 & +0.03 & -0.03 \\
$t \bar{t}+$ light modelling & +0.16 & -0.16 & +0.03 & -0.03 \\
Other background modelling & +0.15 & -0.14 & +0.03 & -0.03 \\
Jet-vertex association, pileup modelling & +0.12 & -0.11 & +0.01 & -0.01 \\
Luminosity & +0.12 & -0.12 & +0.01 & -0.01 \\
Light lepton $(e, \mu)$ ID, isolation, trigger & +0.01 & -0.01 & $<+0.01$ & $<-0.01$ \\
\hline Total systematic uncertainty & +0.72 & -0.79 & +0.13 & -0.11 \\
\hline \hline$t \bar{t}+\geq 1 b$ normalisation & +0.36 & -0.36 & +0.03 & -0.03 \\
$t \bar{t}+\geq 1 c$ normalisation & +0.15 & -0.14 & +0.02 & -0.02 \\
\hline Total statistical uncertainty & +0.44 & -0.43 & +0.08 & -0.08 \\
\hline \hline Total & +0.84 & -0.90 & +0.15 & -0.13 \\
\hline
\end{tabular}

Table 1: Summary of the effects of the systematic uncertainties on $\mu$ for an $H^{+}$signal with a mass of $300 \mathrm{GeV}$ (left) and $800 \mathrm{GeV}$ (right). Due to correlations between the different sources of uncertainties, the total systematic uncertainty can be different from the sum in quadrature of the individual sources. The normalisation factors for both $t \bar{t}+\geq 1 b$ and $t \bar{t}+\geq 1 c$ are included in the statistical component [2]. 
exclusion line reaches $\tan \beta=0.5$ at an $H^{+}$mass value of $948 \mathrm{GeV}$. For $H^{+}$masses around 300 $\mathrm{GeV}$ the most stringent limits on $\tan \beta$ are set, and high values of $\tan \beta$ are excluded in the observed (expected) $\mathrm{H}^{+}$mass range 300-366 (300-378) GeV, the observed exclusion ranging from $\tan \beta>44$ at $300 \mathrm{GeV}$ to $\tan \beta>60$ at $366 \mathrm{GeV}$.
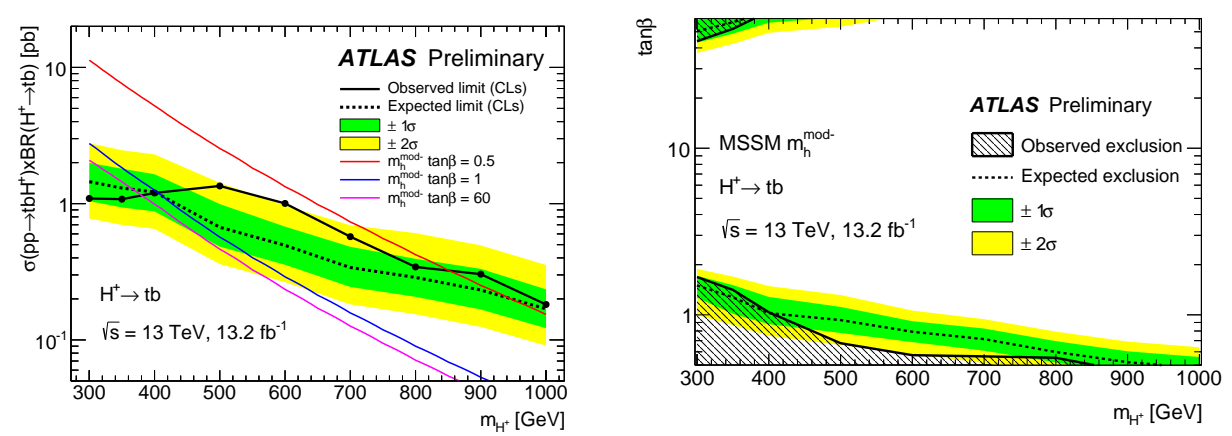

Figure 3: (left) Expected and observed limits for the production of $H^{+} \rightarrow t b$ in association with a top quark and a bottom quark, as well as bands for the $68 \%$ and $95 \%$ confidence intervals; (right) Expected and observed limits on $\tan \beta$ as a function of the charged Higgs boson mass, as well as $68 \%$ and $95 \%$ confidence intervals in the $m_{h}^{\text {mod- }}$ scenario of the MSSM [2].

\section{References}

[1] ATLAS Collaboration, The ATLAS Detector, JINST 3 (2008) S08003.

[2] ATLAS Collaboration, Search for charged Higgs bosons in the $H^{ \pm} \rightarrow$ tb decay channel in pp collisions at $\sqrt{s}=13 \mathrm{TeV}$ using the ATLAS detector, ATLAS-CONF-2016-089, https://cds.cern.ch/record/2206809.

[3] A.L. Read, Presentation of search results: the $C L_{s}$ technique, J.Phys. G28 (2002) 2693.

[4] G. Cowan, K. Cranmer, E. Gross and O. Vitells, Asymptotic formulae for likelihood-based tests of new physics, Eur. Phys. J. C 71 (2011) 1554, arXiv:1007.1727 [physics.data-an].

[5] M. Carena et al., MSSM Higgs Boson Searches at the LHC: Benchmark Scenarios after the Discovery of a Higgs-like Particle, Eur. Phys. J. C 73 (2013) 2552, arXiv:1302.7033 [hep-ph].

[6] LHC Higgs Cross Section Working Group and Heinemeyer et al. editors, Handbook of LHC Higgs Cross Sections: 3. Higgs Properties, CERN-2013-004, arXiv:1307.1347 [hep-ph].

[7] E. Bagnaschi et al. Benchmark scenarios for low $\tan \beta$ in the MSSM, LHCHXSWG-2015-02, https://cds.cern.ch/record/2004747. 